Trinity University

Digital Commons@ Trinity

Mathematics Faculty Research

Mathematics Department

$12-2005$

\title{
The Relationship Between the Number of Shots and the Quality of Gamma Knife Radiosurgeries
}

D Cheek

Allen G. Holder

Trinity University, aholder@trinity.edu

M Fuss

B Salter

Follow this and additional works at: https://digitalcommons.trinity.edu/math_faculty

Part of the Mathematics Commons

\section{Repository Citation}

Cheek, D., Holder, A., Fuss, M., \& Salter, B. (2005). The relationship between the number of shots and the quality of gamma knife radiosurgeries. Optimization and Engineering, 6(4), 449-462. doi:10.1007/s11081-005-2067-1

This Post-Print is brought to you for free and open access by the Mathematics Department at Digital Commons @ Trinity. It has been accepted for inclusion in Mathematics Faculty Research by an authorized administrator of Digital Commons @ Trinity. For more information, please contact jcostanz@trinity.edu. 


\title{
The Relationship Between the Number of Shots and the Quality of Gamma Knife Radiosurgeries
}

\author{
D. Cheek ${ }^{a}$, A. Holder ${ }^{b}$, M. Fuss ${ }^{c}$ and B. Salter ${ }^{d}$
}

April 14, 2004

\begin{abstract}
Radiosurgery is a non-invasive alternative to brain surgery that uses a single focused application of high radiation to destroy intracerebral target tissues. A Gamma Knife delivers such treatments by using 201 cylindrically collimated cobalt- 60 sources that are arranged in a hemispherical pattern and aimed to a common focal point. The accumulation of radiation at the focal point, called a "shot" due to the spherical nature of the dose distribution, is used to ablate (or destroy) target tissue in the brain. If the target is small and spherical, it is easily treated by choosing one of four available collimators $(4,8,14$, or $18 \mathrm{~mm})$. For large, irregular targets, multiple shots are typically required to treat the entire lesion, and the process of determining the optimal arrangement and number of shots is complex.

In this research, fast simulated annealing and a novel objective function are used to investigate the relationship between the number of shots and the quality of the resulting treatment. Sets of $5,10,25,50$, and an unrestricted number of shots are studied for an arteriovenous malformation (AVM). As the shot limit increases the following improvements in plan quality are observed: the conformity of the prescription isodose line increases, the lesion dose becomes more homogeneous, and an increase use of smaller collimators to deposit dose. Large improvements in plan quality are realized by increasing the number of shots from 5 to 50 , and to achieve a similar magnitude of improvement past 50 requires an increase over 1500 shots for the complex lesion investigated. This observation suggests that it is clinically valuable to improve the Gamma Knife's delivery capabilities so that 50 shot treatments are possible.
\end{abstract}

Keywords: stereotactic radiosurgery, Gamma Knife, simulated annealing, conformality index, objective function

a Radiological Sciences University of Texas Health Science Center at San Antonio/ Cancer Therapy and Research Center, San Antonio, TX, cheek@saci.org

${ }^{b}$ Assistant Professor Department of Mathematics, Trinity University, San Antonio, TX, aholder@trinity.edu

c Assistant Professor Department of Radiation Oncology University of Texas Health Science Center, San Antonio, TX, fuss@uthscsa.edu

$d$ Assistant Professor Department of Radiation Oncology University of Texas Health Science Center at San Antonio/ Cancer Therapy and Research Center, San Antonio, TX bsalter@saci.org 


\section{Introduction}

In 1951, Dr. Lars Leksell introduced the concept of radiosurgery, which is the treatment of brain lesions with a single dose of radiation [6]. The term radiosurgery is used because the treatment produces biological effects which are similar to surgical removal. In 1968, the first Gamma Knife was developed to deliver such radiosurgery treatments [7]. In its current configuration, the Gamma Knife uses 201 radioactive cobalt-60 sources that are arranged in a hemispherical pattern and collimated into cylindrical beams that intersect at a common focal point, called the isocenter. Such an arrangement leads to the accumulation of a high radiation dose at the isocenter, and the spherical shape of the dose distribution is called a shot. Four different collimators $(4,8,14$, and $18 \mathrm{~mm})$ are available to adjust the beam diameter, which in turn defines the diameter of the shot.



Figure 1: Gamma Knife cut away. ${ }^{1}$

The Gamma Knife treats many different brain lesions. In general, the goal is to deliver a highly conformal radiation dose with a steep gradient at the edge to spare adjacent healthy tissue. Small, spherically shaped target volumes (typically brain metastases) are easily treated by choosing a collimator $(4,8,14$, or $18 \mathrm{~mm})$, a single isocenter, and an appropriate dose. To conform to a large, irregularly shaped target requires multiple shots with varying isocenters, collimators, and radiation doses (the length of time a source delivers radiation to a shot is called a dose weight). This makes treatment planning a complex process.

The difficulty arises from manually determining the best placement of shots, collimators, and dose weights in a three-dimensional patient image. First attempts are often not acceptable, and the initial shots, collimators, and dose weights are varied to improve the treatment. Professional experience and time constraints limit this trial-and-error approach. The planning process is further complicated by the three-dimensional nature of the problem and the two-dimensional display of the resultant dose distribution. This is because changing a treatment to improve the dose distribution on one 2D image often adversely affects another 2D image.

The number of possible shot locations makes it impossible for a human to consider each of them. For example, if we allow any shot location whose isocenter falls inside a $12.2 \mathrm{~cm}^{3}$ target, then approximately 12 million potential shots exist, assuming a shot is placed every $0.1 \mathrm{~mm}$.

\footnotetext{
${ }^{1}$ Image courtesy of MidMichigan Health, www.midmichigan.org
} 
At each shot location there is a choice of four different collimators and a varying number of dose weights. Due to the size of the solution space, it is difficult to use a trial-and-error process to search for a nearly optimal treatment. Optimization methods represent an alternative that offers the possibility of improving treatments.

Other researchers have designed Gamma Knife treatments with various optimization schemes [5, $13,14,15,16,18,19,20,21]$. Several investigators model the problem as a geometric problem, where optimum shots fall along a skeletonization of the lesion $[1,2,18]$. A modified Powell's method, combined with simulated annealing, is proposed in [19]. Other researchers describe the problem as a constrained, nonlinear programming problem and solve it with the CONOPT optimization software [13].

Several factors are responsible for the variety of approaches. One factor is the amount of time allocated for treatment planning. A patient is typically waiting with a neurosurgically positioned, invasive halo attached to his or her skull while treatment planning is undertaken. This halo cannot be removed from the patient until after treatment, as it establishes a precise coordinate system and restricts patient motion to under a millimeter. This time restriction, along with available computer resources, significantly affects the choice of optimization algorithm, objective function, dose model, and the number of dose calculations. Compromising these can have a significant impact on how accurately the optimization model represents the real problem. Another factor responsible for the variety of approaches is the challenge of capturing the clinical objective(s) within the bounds of the proposed optimization method. Global optimization algorithms are often needed to accommodate the physician's goals, and while there are several global optimization techniques, we use simulated annealing.

The number of shots allowed dramatically affects the treatment delivery time and is desired to be small. For each shot, the patient is removed from the treatment machine and manually positioned to the next location. However, recent advances in technology have made the delivery of multiple shot treatments more efficient by motorizing the movement of the patient from one position to the next. Currently, the motorized movement is performed with the patient removed from the Gamma Knife, but it is foreseeable that these movements will take place while the patient is under treatment. Such a delivery could, theoretically, allow for the delivery of an unlimited number of shots.

In light of the goals and limitations of the Gamma Knife, our aim is to investigate the relationship between the number of shots allowed in a treatment and the quality of the treatment. Because our implementation is intended for research purposes, we are not confined by the previously mentioned clinical time constraints, and we favored accuracy over computational speed. We implement a high precision 3D dose model, a finely spaced dose grid, a novel conformality based objective function, and a fast simulated annealing routine. We investigate the affects of increasing the number of shots on the dose conformality for an irregularly shaped arteriovenous malformation (AVM). Treatments are limited to 5, 10, 25, 50, and an unrestricted number of shots.

\section{A Conformality Based Optimization Model}

Treatment design begins by delineating the lesion (target) to be treated and segmenting the normal tissue on the patient images. These regions are inputs into the treatment planning process. To treat the lesion, a physician or medical physicist selects an appropriate collimator $c$, which controls the diameter of the sphere of dose, a focal point (or isocenter) $f$, and the amount of radiation to deliver. A set of resultant isodose lines (curves that encompasses the volume receiving at least the isodose value) are displayed on the image volume, and the plan is judged. If the coverage of the target is lacking, the procedure is repeated until sufficient coverage and conformality are achieved. Typically, a shot limit $L$ is imposed for reasonable treatment times.

\subsection{Dose Model}

Dose points are placed throughout the patient volume to represent the dose distribution in the images, typically a grid is used. If the grid resolution increases, the accuracy of the calculated 




Figure 2: Conceptual diagram of dose calculation.

dose distribution also increases but at the expense of computer resources. We use Nizin's dose model to calculate how dose from a single source $s$ accumulates at dose point $p$ [10]. We let $a_{(p, f, s, c)}$ be the rate at which point $p$ accumulates radiation from source $s$ as it is restricted by collimator $c$ and directed on isocenter $f$. From [10] we have that if the beams radius $r$ at depth $T D$ is less than $0.35 \mathrm{~cm}$, then

$$
a_{(p, f, c)}=\left(1-e^{-\gamma r}\right) \cdot S_{c}(r) \cdot \operatorname{TAR}(d, r) \cdot \operatorname{OAF}(T D, O A D) \cdot \operatorname{ISF}(S A D, S S D+T D) .
$$

Similarly, if $r \geq 0.35 \mathrm{~cm}$, then

$$
a_{(p, f, c)}=S_{c}(r) \cdot \operatorname{TAR}(d, r) \cdot \operatorname{OAF}(T D, O A D) \cdot \operatorname{ISF}(S A D, S S D+T D) .
$$

The variables of these equations are depicted in figure 2 . In these equations, $T D$ is the depth of the point along the central axis and $O A D$ is the perpendicular distance of the point from the central axis. The constants are the lateral primary dose accommodation coefficient, $\gamma=$ $13.178 \mathrm{~cm}^{-1}$, and $S A D=40 \mathrm{~cm}$. The factor $\left(1-e^{-\gamma r}\right)$ describes the lateral primary dose accrument for field sizes less than $0.35 \mathrm{~cm}$. The function $S_{c}$ characterizes the influence of scatter photons from the collimator, and the function $T A R$ describes the contribution of scatter from the medium to the dose. $O A F$ describes the ratio of dose at the calculation point $p$ to the dose at the central axis at the same depth. The final factor, ISF, models the intensity fall-off of photons with distance from the source. (More information on dose models is found in [4].)

We let $x_{(f, s, c)}$ be the amount of radiation leaving source $s$ with collimator $c$ when the patient is located with focal point $f$. The integral dose at point $p$ from multiple shots is

$$
d_{p}=\sum_{c, f, s} a_{(p, f, s, c)} x_{(f, s, c)}, \forall p
$$

Since each source delivers the same amount of radiation per shot, we define $a_{(p, f, c)}$ to be $\sum_{s} a_{(p, f, s, c)}$. Allowing $x_{(f, c)}$ to be the amount of radiation delivered by each source in shot $(f, c)$, we have that

$$
d_{p}=\sum_{c, f} a_{(p, f, c)} x_{(f, c)}, \forall p .
$$

We point out that dose deposition is a linear function of $x$.

\subsection{The Optimization Model}

A scoring metric is valuable for ranking treatments, as multiple plans are generated and the most clinically acceptable one is delivered. The designation of most acceptable is often decided 
by the one that best conforms to the lesion, as quantified by a conformality index. One such index is the Ian Paddick conformality index (IPCI) [12].

$I P C I$ quantifies the conformity of an isodose line to the lesion surface. It is composed of two components: the over treatment ratio $(O T R)$ and the under treatment ratio $(U T R)$, where $0 \leq O T R \leq 1$ and $0 \leq U T R \leq 1$. A perfect score of 1 for $O T R$ implies there is no over treatment of normal tissue and a perfect score of 1 for $U T R$ implies there is no under treatment of the target. The OTR and UTR are defined by the volumes of tissue in the target and in an isodose line, and these volumes are measured by the number of dose points they contain. We define

$$
\begin{aligned}
T V & =\{p: \text { dose point } p \text { is in the target }\} \text { and } \\
I V_{T} & =\left\{p: d_{p} \geq T \cdot D\right\}
\end{aligned}
$$

where $T$ is a percentage of the prescribed tumor dose $D$. If each dose point represents a volume $V$, then $V|T V|$ and $V\left|I V_{T}\right|$ are the target volume and the isodose volume, respectively. The definitions of $O T R, U T R$, and IPCI are,

$$
\begin{aligned}
O T R_{T} & =\frac{\left|T V \cap I V_{T}\right|}{\left|I V_{T}\right|}, \\
U T R_{T} & =\frac{\left|T V \cap I V_{T}\right|}{|T V|}, \text { and } \\
I P C I_{T} & =O T R \times U T R=\frac{\left(\left|T V \cap I V_{T}\right|\right)^{2}}{|T V| \times\left|I V_{T}\right|} .
\end{aligned}
$$

Figure 3 depicts a grid of dose points with a lesion partially to fully encompassed by three isodose lines, $100 \%, 70 \%$, and $50 \%$. From figure 3 we see the $100 \%$ isodose line encompasses 26 points; 21 of 24 lesion points and 5 normal tissue points. Therefore, $O T R_{100 \%}=\frac{21}{26}=0.808$, $U T R_{100 \%}=\frac{21}{24}=0.875$, and $I P C I_{100 \%}=0.808 \times 0.875=0.707$.



Figure 3: Conceptual diagram of IPCI. 100\%, 70\%, and 50\% isodose lines displayed with a grid of dose points.

We adopt the philosophy to optimize the metric that is often used to judge a plan. From figure 3 we see that the $100 \%$ isodose line has a higher degree of conformality than the $70 \%$ and $50 \%$ isodose lines. To accurately model the physician's objectives, namely to treat only the lesion, we want to maximize the $I P C I_{100 \%}, I P C I_{70 \%}$, and $I P C I_{50 \%}$. This forces a steep dose gradient at the edge of the high dose region. However, we have found that equally weighting the IPCIs causes under dosage of the lesion at the edges. This is not consistent with the physician's desire to treat the entire lesion to the prescribed, or $100 \%$, isodose level. Therefore, careful weighting of each IPCI is important.

As previously stated, the treatment of a large, nonspherical lesion often requires the placement of multiple shots with different focal points. To adequately cover the lesion, the spherical high dose regions typically overlap, producing a high dose inhomogeneity, or hot spot, that produces radiation levels higher than what was prescribed. There are two viewpoints within the community about the clinical impact of such inhomogeneities. One perspective is that hot spots are desirable toward the ablative intent of radiosurgery. The other belief links hot spots with complications and/or toxicity [9]. We incorporate a linear penalty to control dose inhomogeneities. In order to quantify inhomogeneities over regions containing several dose points, we 
let $\left\{H_{r}: r=1,2, \ldots, R\right\}$ be a partition of the dose points, where each $H_{r}$ defines a region over which dose is aggregated. The complete model is

Minimize:

$$
E=\sum_{i=1} w_{i}\left(1-\frac{\sum_{p \in T V} \alpha_{(i, p)}}{\sum_{p=1}^{P} \alpha_{(i, p)}}\right)+\sum_{i=1} u_{i}\left(1-\frac{V}{K} \sum_{p \in T V} \alpha_{(i, p)}\right)+h \cdot q
$$

Subject to:

$$
\begin{array}{rlrl}
d_{p} & =\sum_{c, f} a_{(p, f, c)} x_{(f, c)}, \forall p & & \text { (calculate dose) } \\
d_{p} & \leq T_{i} D+\alpha_{(i, p)} M, \forall i, p & & \text { (calculate isodose volumes) } \\
x_{(f, c)} & \leq \beta_{(f, c)} M, \forall f, c & & \text { (calculate the number of shots) } \\
\sum_{c, f} \beta_{(f, c)} & \leq L & & \text { (bound the number of shots) } \\
\frac{1}{\left|H_{r}\right|} \sum_{p \in H_{r}} d_{p} & \leq \tau+q, \forall r & & \\
\sum_{p=1}^{P} \alpha_{(i, p)} & \geq 1, \forall i & & \text { (measure hot spot in region } H_{r} \text { ) } \\
x & \geq 0 & & \\
\alpha_{(i, p)} & \in\{0,1\} & & \\
\beta_{(f, c)} & \in\{0,1\} . &
\end{array}
$$

Note, we do not optimize IPCI directly, but instead consider a weighted sum of OTR and UTR. This allows greater flexibility in controlling tradeoffs between $O T R$ and $U T R$. The weights $w_{i}$ and $u_{i}$ decide the importance of $O T R$ and $U T R$ for each isodose line $i$. The parameters $V$ and $K$ are the voxel and target volumes, respectively, and $h$ penalizes the maximum dose inhomogeneity above the threshold $\tau$. The isodose lines are defined by the thresholds $T_{i}$, and the maximum number of shots is $L$. The value of $M$ is arbitrarily large.

In this research, we maintained all the model parameters as constants except for $L$ in order to investigate how limiting the number of shots effects the dose distribution. However, in a clinical situation a physician or medical physicist could modify any of the parameters to more accurately model the treatment goals of a particular patient.

\subsection{Optimization Routine}

We use fast-simulated annealing to approximately solve our optimization problem (see [3, 17] for a complete description). An iteration from $x^{k}$ to $x^{k+1}$ is made by selecting a focal spot $f^{\prime}$, a collimator $c^{\prime}$, and an amount of radiation change $y$ for the 201 sources,

$$
x_{(f, c)}^{k+1}=\left\{\begin{aligned}
x_{\left(f^{\prime}, c^{\prime}\right)}^{k}+y, & (f, c)=\left(f^{\prime}, c^{\prime}\right) \\
x_{(f, c)}^{k}, & \text { otherwise, }
\end{aligned}\right.
$$

where $f^{\prime}$ and $c^{\prime}$ are uniformly distributed and $y$ is selected according to the Cauchy distribution with mean 0 . The annealing schedule and the variance of $y$ are cooled by

$$
T_{n}(k)=\frac{T_{0}^{n}}{1+\frac{k}{B_{n}}},
$$




\begin{tabular}{|c||c|c|c|}
\hline$i$ & Isodose Line & $w_{i}$ & $u_{i}$ \\
\hline \hline 1 & $100 \%$ & 5 & 5 \\
\hline 2 & $50 \%$ & 0.5 & 0.5 \\
\hline \hline
\end{tabular}$\quad$\begin{tabular}{|c|c|c|}
\hline & & $h$ \\
\hline \hline $100 \%$ & 0.5 \\
\hline
\end{tabular}

Table 1: Objective function parameters.

where $T_{0}$ is the initial temperature, $B$ is a user defined constant, and $n$ indicates whether or not we are cooling the variance of $y(n=1)$ or the annealing schedule $(n=2)$.

An iteration is automatically accepted if the objective $E^{k+1}$ is less than $E^{k}$ (the index corresponds with the above iterations). Otherwise, we randomly generate a $\beta$ and accept the move if

$$
\beta \leq e^{-\left(E_{k}-E_{k+1}\right) / T_{2}(k)} .
$$

The stopping criteria for the optimization is when $k \approx 9 \times 10^{6}$. On a 2 gigahertz computer with 1 gigabyte of RAM, a single optimization takes approximately 10 days.

\subsection{Overall Experiment Design}

The Gamma Knife has the ability to deliver shots at a $0.1 \mathrm{~mm}$ resolution. Therefore, for a $12.2 \mathrm{~cm}^{3}$ lesion there are approximately 12 million potential shot locations in the lesion. Modern computers are not capable of storing this large number of potential shot locations. For example, if each shot requires 50 kilobytes of RAM, then approximately 600 Gigabytes of RAM are needed. To overcome this computational issue, we divide the design process into multiple steps. First, shots are spaced at $2 \mathrm{~mm}$ intervals and our optimization model is solved. Once the first optimization is complete, we refine the grid around each optimal shot in order to more accurately locate optimal isocenters. The process is repeated until the resolution is $0.5 \mathrm{~mm}$. Our current technique refines the grid by halving the distance, so the first isocenters are placed on a $2 \mathrm{~mm}$ grid, the second isocenters are located on a $1 \mathrm{~mm}$ grid, and the third isocenters are on a $0.5 \mathrm{~mm}$ gird. This multi-step optimization allows us to work under current RAM limitations. Since the model is solved three times, the computational time for a single plan with $0.5 \mathrm{~mm}$ resolution is approximately 30 days.

The user definable arguments for this study are displayed in Table 1. We chose two isodose levels in our model; the $100 \%$ and $50 \%$. The $100 \%$ and $50 \%$ isodose lines are weighted with a 10 and 1, respectively, in order to appreciate the conformality of the $100 \%$ isodose line over the $50 \%$ isodose line. The value of $h$ is small at 0.5 , in order that the optimization consider the value of reducing hot spots but not at the expense of conformality.

\section{Results}

Figure 4 displays the $100 \%, 70 \%, 50 \%$, and $30 \%$ isodose lines, normalized to the prescription dose, on the patient images for the five treatments designed by our model. Observe that the $100 \%$ isodose line conforms to the lesion as the shot limit is increased.

The dose-volume histograms (DVHs) in Figure 5 capture the $3 \mathrm{D}$ dose distribution in a single graph and are useful when evaluating radiosurgery plans. These accumulative graphs fail to represent spatial information but accurately quantify the volume of a structure that receive above a specified dose. The physician's ideal DVH is a step function that shows that $100 \%$ of the lesion receives precisely the prescribed dose. Figure 5 demonstrates that the DVH for the lesion approaches the physician's ideal DVH as the number of shots is increased. The most noticeable effect on the DVH for the lesion is the dramatic decrease in the tail portion with increased numbers of shots, which signifies an increase in dose homogeneity. There are two widely held beliefs in the radiosurgery community. One being that high dose inhomogeneity (i.e. hot spots) in the lesion is consistent with the physician's ablative intent for the lesion and, therefore, poses no real problem. The other is that a high dose inhomogeneity could potentially lead to complications/toxicity and is therefore undesirable. For the purposes of characterizing 


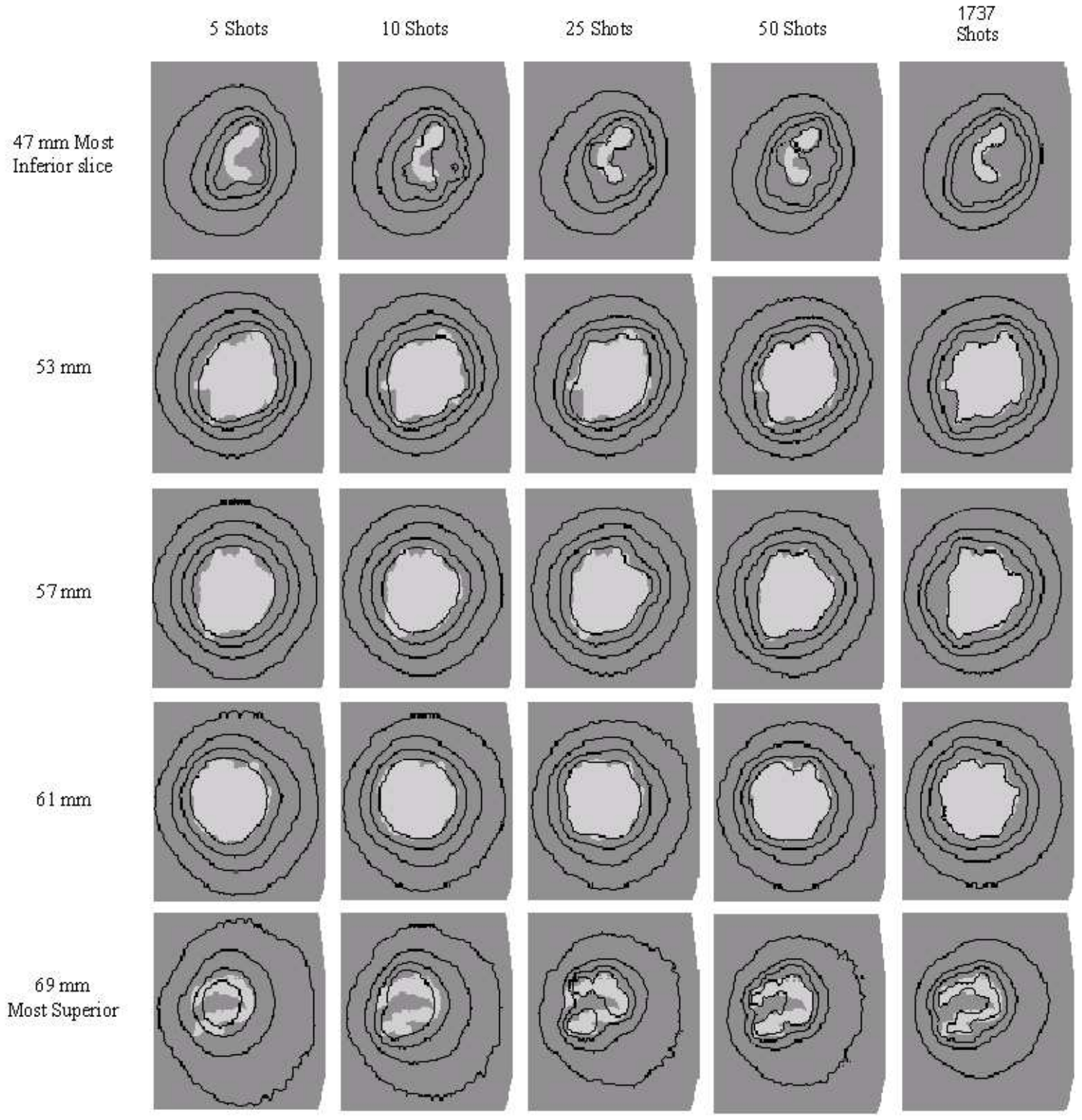

Figure 4: Axial isodose distributions for the five optimized plans. $100 \%, 70 \%, 50 \%$, and $20 \%$ isodose lines depicted. 




Figure 5: Lesion dose-volume histogram comparison of the five optimized plans

the quality of a treatment in our research, homogeneity of dose in the lesion is interpreted as a desirable endpoint, which is why $h$ is positive.

The statistics are summarized in Table 2, and as the number of shots increases, there is a progression toward improved treatment plans. As an example, the $I P C I_{100 \%}$ goes from 0.767 when only 5 shots are allowed to an almost perfect score of 0.995 when the number of shots is unrestricted. This is supported by Figure 4 because the $100 \%$ isodose line conforms to the lesion as the number of shots is increased. Notice that the $100 \%$ isodose line transitions from a roughly spherical shape in the five shot plan, much like a single shot, to a non-spherical shape when the number of shots is unlimited.

We might expect that all of the clinically significant isodose lines would become more conformal as the number of shots increases. However, the unrestricted shot case shows that the $70 \%$ isodose line is less conformal than the other $70 \%$ isodose lines. The normal tissue DVH in Figure 6 shows that the unrestricted plan is actually inferior to the other four plans for the $65 \%$ and $85 \%$ isodose levels. This is the result of the objective function having knowledge of only the $100 \%$ and $50 \%$ isodose lines in our implementation. The addition of a $75 \%$ isodose line would likely resolve the issue.

How the different collimators are used in the optimized plans is depicted in Figure 7. Intuition suggests that a higher-quality dose distribution is possible by using the smallest collimator and 


\begin{tabular}{|c||c|c|c|c|c|c|}
\hline & 5 Shots & 10 Shots & 25 Shots & 50 Shots & $\begin{array}{c}1737 \\
\text { Shots }\end{array}$ & $\begin{array}{c}\text { Ideal } \\
\text { Value }\end{array}$ \\
\hline \hline$I P C I_{100 \%}$ & 0.767 & 0.808 & 0.863 & 0.919 & 0.995 & 1 \\
\hline$O T R_{100 \%}$ & 0.846 & 0.897 & 0.925 & 0.954 & 0.997 & 1 \\
\hline$U T R_{100 \%}$ & 0.906 & 0.901 & 0.932 & 0.963 & 0.998 & 1 \\
\hline $\mathrm{E}$ & 0.155 & 0.128 & 0.099 & 0.069 & 0.029 & 0 \\
\hline $\begin{array}{l}\text { Maximum 0.064 cm } \mathrm{cm}^{3} \text { Volu- } \\
\text { metric Hot Spot Normalized } \\
\text { to Prescription Dose }\end{array}$ & 3.218 & 2.723 & 2.472 & 2.013 & 1.107 & 1 \\
\hline $\begin{array}{l}\text { Amount of Normal Tissue } \\
\text { above the Prescription Dose } \\
\text { in } \mathrm{cm}^{3}\end{array}$ & 2.016 & 1.272 & 0.92 & 0.568 & 0.04 & 0 \\
\hline
\end{tabular}

Table 2: Statistics of the five optimized plans.

an increased number of shots. Figure 7 supports our intuition. In the five shot plan, $81 \%$ of the integral dose is deposited by the 14 and $18 \mathrm{~mm}$ collimators. Conversely, in the unrestricted plan the 14 and $18 \mathrm{~mm}$ collimators deposit only $2 \%$ of the integral dose and the $4 \mathrm{~mm}$ collimator deposits $78 \%$ of the integral dose. Thus, for this irregularly shaped target, the smaller $4 \mathrm{~mm}$ collimator is more useful than the larger $14 \mathrm{~mm}$ and $18 \mathrm{~mm}$ collimators as the number of shots is increased.

Finally, the results suggest a more subtle conclusion beyond the recognition that increased numbers of shots leads to improvements in plan quality. To achieve a similar magnitude of improvement as from 5 to 50 shots requires an increase over 1500 shots. Currently technology can allow the timely delivery of 25 to 50 shots. It is foreseeable that a moving shot delivery methodology, where the patient is not removed to set the shot coordinates, could allow the delivery of an unlimited number of shots. Thus, improving the treatment quality to an almost ideal plan.

\section{Conclusion}

We investigated the relationship between the number of shots allowed in a treatment and the quality of the resulting radiosurgery plan for a discrete set of $5,10,25,50$, and an unlimited number of shots. A novel Ian Paddick conformality based objective function was solved via fast simulated annealing, keeping all parameters constant except the shot limitation $L$.

As $L$ increased the following improvements in plan quality were observed for the AVM: the conformity of the prescription isodose line increased, the lesion dose became more homogeneous, and an increased use of smaller collimators to deposit dose. Overall, the plan quality approached the physician's ideal as $L$ increased.

Currently, the Gamma Knife does not have the capability to deliver an unlimited number of shots. The manufacturer has recently motorized the patient movement, but they remove the patient from the machine to set the coordinates. It is foreseeable the motorized coordinate change will take place without removal, thus enabling a moving shot delivery of an unlimited number of shots. In the future we plan to investigate the clinical feasibility of such a methodology.

\section{Acknowledgments}

We thank Dr. Paul Nizin and Dr. Rob Mooij for their support via valuable email interactions. We also thank the careful reading and suggestions of the two anonymous reviewers. 


\section{Dose-Volume Histogram of Normal Tissue}



Figure 6: Partial DVH of normal tissue for the five optimized plans.

\section{References}

[1] M. Ferris, J. Lim, and D. Shepard. An optimization approach for the radiosurgery treamtment planning. SIAM Journal on Optimization, 13(3):921-937, 2003.

[2] M. Ferris, J. Lim, and D. Shepard. Radiosurgery optimization via nonlinear programming. Annals of Operations Research, 119:247-260, 2003.

[3] S. Geman and D. Geman. Stochastic relaxation, gibbs distribution, and the bayesian restoration of images. IEEE Transactions on Pattern Analysis and Machine Intelligence, 6:721-741, 1984 .

[4] Faiz M. Khan. The Physics of Radiation Therapy. Lippincott Williams and Wilkins, 1994.

[5] G. Leichtman, A. Aita, and H. Goldman. Automated gamma knife dose planning using polygon clipping and adaptive simulated annealing. Medical Physics, 27(1):154-162, 2000.

[6] L. Leksell. The stereotactic method and radiosurgery of the brain. Acta Chirurgica Scandinavica, 102:316-319, 1951.

[7] L. Leksell. Cerebral radiosurgery. Acta Chirurgica Scandinavica, 134(8):585-595, 1968.

[8] D McShan and B Fraass. Use of an octree-like geometry for 3-d dose calculations. Medical Physics, 20(4):1219-1227, 1993.

[9] L. Nedzi, K. Hanne, E. Alexander III, R. Gelman, and J. Loeffle. Variables associated with the development of complications from radiosurgery of intracranial tumors. International Journal of Radiation Oncology, Biology, and Physics, 21(3):591-599, 1991.

[10] P. Nizin. On absorbed dose in narrow ${ }^{60} \mathrm{Co}$ gamma-ray beams and dosimetry of the gamma knife. Medical Physics, 25(12):2347-2351, 1998. 




Number of Allowed Shots

Figure 7: Percent of integral dose deposited by respective collimators for the five optimized plans.

[11] P. Nizin and R. Mooij. Tissue-air ratios for narrow ${ }^{60} \mathrm{Co}$ gamma-ray beams. Medical Physics, 25(8):1458-1463, 1998.

[12] I. Paddick. A simple scoring ratio to index the conformity of radiosurgical treatment plans. Journal of Neurosurgery, 93(3):219-222, 2000.

[13] D. Shepard, M. Ferris, R. Ove, and L. Ma. Inverse treatment planning for gamma knife radiosurgery. Medical Physics, 27(12):2748-2756, 2000.

[14] H. Shu, Y. Yan, X. Bao, Y. Fu, and L. Luo. Treatment planning optimization by quasinewton and simulated annealing methods for gamma unit treatment system. Physics in Medicine and Biology, 43(10):2795-2805, 1998.

[15] H. Shu, Y. Yan, L. Luo, and X. Bao. Three-dimensional optimization of treatment planning for gamma unit treatment system. Medical Physics, 25(12):2352-2357, 1998.

[16] L. Luo H. Shu, W. Yu, Y. Yan, X. Bao, and Y. Fu. Optimizing computerized treatment planning for the gamma knife by source culling. International Journal of Radiation Oncology Biology and Physics, 45(5):1339-1346, 1999.

[17] H. Szu and R. Hartley. Fast simulated annealing. Physics Letters A, 122(3-4):157-162, 1987.

[18] Q. Wu and J. Bourland. Morphology-guided radiosurgery treatment planning and optimization for multiple isocenters. Medical Physics, 26(10):2151-2160, 1999.

[19] Y. Yan, H. Shu, X. Bao, L. Luo, and Y. Bai. Clinical treatment planning optimization by powell's method for gamma unit treatment system. International Journal of Radiation Oncology, Biology, and Physics, 39(1):247-254, 1997. 
[20] P. Zhang, D. Dean, A. Metzger, and C. Sibata. Optimization o gamma knife treatment planning via guided evolutionary simulated annealing. Medical Physics, 28(8):1746-1752, 2001.

[21] P. Zhang, J. Wu, D. Dean, L. Xing, J. Xue, R. Maciunas, and C. Sibata. Plug pattern optimization for gamma knife radiosurgery treatment planning. International Journal of Radiation Oncology, Biology, and Physics, 55(2):420-427, 2003. 